\title{
A FILTRATION ON EQUIVARIANT BOREL-MOORE HOMOLOGY
}

\author{
ARAM BINGHAM ${ }^{1}$, MAHIR BILEN CAN ${ }^{1}$ and YILDIRAY OZAN ${ }^{2}$ \\ ${ }^{1}$ Department of Mathematics, Tulane University, New Orleans, 70118, LA, USA; \\ email: abingham@tulane.edu, mahirbilencan@gmail.com \\ ${ }^{2}$ Department of Mathematics, Middle East Technical University, Ankara, Turkey; \\ email: ozan@metu.edu.tr
}

Received 13 September 2018; accepted 2 June 2019

\begin{abstract}
Let $G / H$ be a homogeneous variety and let $X$ be a $G$-equivariant embedding of $G / H$ such that the number of $G$-orbits in $X$ is finite. We show that the equivariant Borel-Moore homology of $X$ has a filtration with associated graded module the direct sum of the equivariant Borel-Moore homologies of the $G$-orbits. If $T$ is a maximal torus of $G$ such that each $G$-orbit has a $T$-fixed point, then the equivariant filtration descends to give a filtration on the ordinary Borel-Moore homology of $X$. We apply our findings to certain wonderful compactifications as well as to double flag varieties.
\end{abstract}

2010 Mathematics Subject Classification: 14C15 (primary); 14M27 (secondary)

\section{Introduction}

Let $G$ be a connected complex algebraic group and let $K$ be a closed subgroup. A $G$-variety $X$ is said to be a $G$-equivariant embedding of $G / K$ if there is an open $G$ orbit $O$ in $X$ such that the stabilizer subgroup of a point from $O$ is isomorphic to $K$. In this paper, we are concerned with the (equivariant) Borel-Moore homology groups of $X$ under the assumption that $X$ contains only finitely many $G$-orbits. Let us denote by $A_{*}^{G}(X)_{\mathbb{Q}}$ the rational $G$-equivariant Chow group of $X$ and by $H_{*}^{G}(X, \mathbb{Q})$ the rational $G$-equivariant Borel-Moore homology group of $X$. Then there is a degree doubling cycle map $c l_{X}^{G}: A_{*}^{G}(X)_{\mathbb{Q}} \rightarrow H_{*}^{G}(X, \mathbb{Q})$; see [11]. In this notation, the main general result of our paper is the following.

(c) The Author(s) 2019. This is an Open Access article, distributed under the terms of the Creative Commons Attribution licence (http://creativecommons.org/licenses/by/4.0/), which permits unrestricted re-use, distribution, and reproduction in any medium, provided the original work is properly cited. 
THEOREM 1. Let $G$ be a complex connected linear algebraic group and let $X$ be a complex $G$-variety. If $X$ comprises finitely many $G$-orbits, then $c l_{X}^{G}$ is an isomorphism between $A_{*}^{G}(X)_{\mathbb{Q}}$ and $H_{*}^{G}(X, \mathbb{Q})$. Furthermore, as a $H_{G}^{*}(p t, \mathbb{Q})$-module, the equivariant Borel-Moore homology has a decomposition, $H_{*}^{G}(X, \mathbb{Q}) \cong H_{*}^{G}(Y, \mathbb{Q}) \oplus H_{*}^{G}(U, \mathbb{Q})$, where $Y$ is any closed orbit in $X$ and $U$ is the complement of $Y$ in $X$. Consequently, the equivariant Borel-Moore homology of $X$ has a filtration, as a graded module over $H_{G}^{*}(p t, \mathbb{Q})$, with associated graded module equal to the direct sum of equivariant Borel-Moore homologies of the $G$-orbits in X.

In our second result, we make an additional assumption so that we have an analogous result for the nonequivariant Borel-Moore homology groups.

THEOREM 2. Let $X$ and $G$ be as in Theorem 1 and let $T$ be a maximal torus of $G$. If each $G$-orbit in $X$ contains a $T$-fixed point, then $X$ has zero cohomology in odd degree. Furthermore, the Borel-Moore homology of $X$ has a filtration as a graded $\mathbb{Q}$-vector space with associated graded space equal to the direct sum of Borel-Moore homologies of G-orbits.

A normal irreducible algebraic variety $X$ on which a connected reductive algebraic group $G$ acts with a morphism $G \times X \rightarrow X$ is called a spherical $G$ variety if a Borel subgroup $B$ of $G$ has an open orbit in $X$. It is well known that a spherical variety $X$ has only finitely many $G$-orbits in it; see [13]. In fact, $X$ is a spherical $G$-variety if and only if there are only finitely many $B$ orbits in $X$; see $[4,17]$. Therefore, Theorem 1 is applicable to any spherical $G$ variety. A particularly interesting subset of the set of spherical varieties consists of wonderful varieties, defined by the following properties:

(1) $X$ is a smooth projective $G$-variety with an open $G$-orbit $X_{0}$;

(2) $X \backslash X_{0}=\bigcup_{i=1}^{l} D_{i}$, where $D_{i}$ 's are smooth prime $G$-divisors with normal crossings and $\bigcap_{i=1}^{l} D_{i} \neq \emptyset$;

(3) if $x$ and $x^{\prime}$ are two points from $X$ such that $\left\{i: x \in D_{i}\right\}=\left\{i: x^{\prime} \in D_{i}\right\}$, then $G \cdot x=G \cdot x^{\prime}$.

The divisors $D_{i}(1 \leqslant i \leqslant l)$ are called the boundary divisors. Note that the intersection of all boundary divisors is a closed subset of $X$; therefore, it is isomorphic to a flag variety $G / Q$ for some parabolic subgroup $Q$ in $G$.

Our third result is an application of the previous theorem. 
THEOREM 3. Let $H$ be a closed subgroup of $G$ such that $G / H$ is isomorphic to the open $G$-orbit in a wonderful variety $X$. Let $\left\{Y_{0} \cong G / H, Y_{1}, \ldots, Y_{r}\right\}$ be the set of all $G$-orbits in $X$, where the closed orbit $Y_{r}$ is isomorphic to the flag variety $Y_{r} \cong G / Q$ for some parabolic subgroup $Q$. If the ranks of $G$ and $H$ are equal, then there is a filtration of the Borel-Moore homology $H_{*}(X, \mathbb{Q})$, as a graded module over $\mathbb{Q}$, with the associated graded module equal to the direct sum of Borel-Moore homology groups of the G-orbits in X.

Let $G$ be a connected reductive group, let $T$ be a maximal torus in $G$, and let $B$ be Borel subgroup such that $T \subset B$. Let $Q$ be a parabolic subgroup containing $B$. It is well known that the cohomology ring of a (partial) flag variety $G / Q$ is isomorphic to the $W_{Q}$-invariants in the ring $H^{*}(G / B)$, where $W_{Q}$ is the Weyl group of (a Levi factor of) $Q$. Another application of our theorem concerns the tensor products of vector spaces of the form $H^{*}(G / Q, \mathbb{Q})$. Let $W$ denote the Weyl group, $N_{G}(T) / T$, and let $\Delta$ denote the set of simple roots determined by $(B, T)$. Let $I$ and $J$ be two subsets from $\Delta$. We denote by $P_{I}$ (respectively by $P_{J}$ ) the parabolic subgroup corresponding to $I$ (respectively to $J$ ), and we denote by $W_{I}$ (respectively by $W_{J}$ ) the Weyl group of $P_{I}$ (respectively of $P_{J}$ ). The Weyl groups $W_{I}$ and $W_{J}$ are subgroups of $W$. For $w$ in $W$, let us denote by $\dot{w}$ an element of $N_{G}(T)$ representing $w$. Finally, let us denote by ${ }^{I} W^{J}$ the subset of $W$ consisting of elements $w \in W$ such that if $v \in W_{I} w W_{J}$, then the minimum number of simple reflections required to write $v$ as a product is greater than or equal to that of $w$.

THEOREM 4. Let $P_{I}$ and $P_{J}$ be two standard parabolic subgroups of a connected reductive subgroup $G$, and let $X$ denote the double flag variety $X:=G / P_{I} \times$ $G / P_{J}$ on which $G$ acts diagonally. Then every $G$-orbit in $X$ contains a $T$ fixed point, and, furthermore, as a $\mathbb{Q}$-vector space, the cohomology ring of $X$ decomposes into a direct sum of cohomology rings as follows:

$$
H^{*}\left(G / P_{I} \times G / P_{J}, \mathbb{Q}\right) \cong \bigoplus_{w \in^{I} W^{J}} H^{*}\left(G / \operatorname{Stab}_{G}(\dot{w}), \mathbb{Q}\right)
$$

The structure of our paper is as follows. In Section 2, we summarize some basic properties of the equivariant Chow groups and the equivariant Borel-Moore homology groups. In Sections 3-5, we prove the theorems that are stated above.

\section{Notation and preliminaries}

Throughout this paper, $G$ will stand for a connected algebraic group defined over $\mathbb{C}$. We will often assume that $G$ is reductive, and we will use the letter $B$ to denote a Borel subgroup of $G$. We will use $T$ to denote a maximal torus that is 
contained in $B$. Then $B=T \ltimes U$, where $U$ is the unipotent radical of $B$. We will denote the Weyl group of $(G, T)$ by the letter $W$. Then $W=N_{G}(T) / T$, where $N_{G}(T)$ is the normalizer of $T$ in $G$.

2.1. (Equivariant) Chow groups. The equivariant Chow groups and the equivariant Borel-Moore homology of a $G$-space are introduced by Edidin and Graham in [11]. In [5], by focusing on the torus actions, Brion constructed the machinery for the applications of equivariant Chow groups. Among the results of Brion is a presentation of the equivariant Chow groups via invariant cycles, which establishes a connection between the equivariant Chow groups and the ordinary Chow groups. We will provide more details for this fact in the sequel.

To set up our notation, we will briefly review the definitions and basic properties of the equivariant Chow groups and Borel-Moore homology groups. We will follow the notation of [6]; however, we will not abbreviate $H_{*}^{G}(X, \mathbb{Q})$ to $H^{G}(X)$.

Let $X$ be a complex algebraic $G$-scheme, where $G$ is a connected complex linear algebraic group $G$. Note that we do not assume the reductiveness of $G$; however, implicit in the definition of a $G$-scheme is the assumption that it has a covering by $G$-invariant quasiprojective open sets. Let $n$ be a nonnegative integer. To define the $k$ th $G$-equivariant Chow group of $X$, we fix a linear representation $V$ of $G$ and a $G$-stable open subset $U$ in $V$ satisfying the following two conditions:

(1) the quotient $U \rightarrow U / G$ exists, and it is a principal $G$-bundle;

(2) $k \leqslant \operatorname{codim}_{V}(V \backslash U)$.

Then the quotient of the product $X \times U$ by the diagonal action of $G$ exists as a scheme, which is denoted by $X \times{ }_{G} U$. If $X$ is equidimensional, then the $G$ equivariant Chow group of degree $k$ of $X$ is defined by

$$
A_{G}^{k}(X):=A^{k}\left(X \times_{G} U\right) .
$$

If $X$ is not necessarily equidimensional, then we put

$$
A_{k}^{G}(X):=A_{k-\operatorname{dim}(G)+\operatorname{dim}(U)}\left(X \times_{G} U\right) .
$$

Obviously, if $X$ is equidimensional, then $A_{G}^{k}(X)=A_{\operatorname{dim}(X)-k}^{G}(X)$.

The $k$ th $G$-equivariant Borel-Moore homology of $X$ is defined by

$$
H_{k}^{G}(X):=H_{k-2 \operatorname{dim}(G)+2 \operatorname{dim}(U)}\left(X \times_{G} U\right) .
$$

The rational equivariant Borel-Moore homology groups as well as the rational equivariant Chow groups are defined, as in the ordinary case, by tensoring the corresponding groups with $\mathbb{Q}$. 
REMARK 7. We will mention several important results that are of crucial importance for our purposes.

(1) The (degree doubling) cycle map between the ordinary Chow groups and Borel-Moore groups lifts to the equivariant setup, $c l_{X}^{G}: A_{k}^{G}(X)_{\mathbb{Q}} \rightarrow$ $H_{2 k}^{G}(X, \mathbb{Q})$.

(2) Let $G$ be a connected reductive complex algebraic group, and let $S$ denote the symmetric algebra over the rationals of the character group of a maximal torus $T$ of $G$. We denote by $W$ the Weyl group of $(G, T)$. In this notation, we have the equality $A_{G}^{*}(p t)_{\mathbb{Q}}=S^{W}$; see [6, Theorem 10]. Also, we see from Lemma 10 that if $P$ is a parabolic subgroup of $G$ with a Levi subgroup $L \subset P$ such that $T \subset L$, then $A^{*}(G / P)_{\mathbb{Q}}=S^{W_{L}} /\left(S_{+}^{W}\right)$, where $W_{L}$ is the Weyl group of the pair $(L, T)$. Since $W_{L} \subset W$, we see that $A^{*}(G / P)_{\mathbb{Q}}$ has the structure of a $A_{G}^{*}(p t)_{\mathbb{Q}}$-module. In fact, for any $G$-scheme $X$, the $G$-equivariant Chow group $A_{*}^{G}(X)$ is a graded module over $A_{*}^{G}(p t)$. These statements remain true if we replace the equivariant Chow rings with the equivariant cohomology rings.

(3) Let $X$ be a $G$-scheme, where $G$ is as in the previous item. It is shown in [6, Proposition 11] that there is a canonical isomorphism $\rho_{G}: A_{*}^{G}(X)_{\mathbb{Q}} /\left(S_{+}^{W}\right) \rightarrow A_{*}(X)_{\mathbb{Q}}$.

(4) If $Y$ is a homogeneous variety of the form $G / K$ for some closed subgroup $K$ of $G$, then the equivariant cycle map $c l_{Y}^{G}: A_{*}^{G}(Y)_{\mathbb{Q}} \rightarrow H_{*}^{G}(Y, \mathbb{Q})$ is an isomorphism. Indeed, it is easy to see from definitions, by using the homotopy invariance property, that $A_{*}^{G}(G / K)_{\mathbb{Q}}=A_{*}^{K}(p t)_{\mathbb{Q}}=A_{*}(B K)_{\mathbb{Q}}$ and that $H_{*}^{G}(G / K, \mathbb{Q})=H_{*}^{K}(p t, \mathbb{Q})=H_{*}(B K, \mathbb{Q})$. Here, $B K$ denotes the classifying space of $K$. The ordinary cycle map $c l_{B K}: A_{*}(B K)_{\mathbb{Q}} \rightarrow$ $H_{*}(B K, \mathbb{Q})$ is an isomorphism. This fact is proven by Edidin and Graham in [10] by building on Totaro's work [15]. Since the cycle map is degree doubling, it also follows from this analysis that $Y$ has zero equivariant Borel-Moore homology in odd degrees.

\section{Proof of Theorem 1}

Proof of Theorem 1. We start with a quick reduction argument since we do not assume that $G$ is reductive. Let $R_{u}(G)$ denote the unipotent radical of $G$ and let $G^{\prime}$ denote the reductive group $G / R_{u}(G)$. Then, for an open set $U$ as in the definition of the $G$-equivariant Chow groups (see (5)), we consider the quotient $U \rightarrow U / G^{\prime}$. This quotient exists, and, furthermore, it is a principal $G^{\prime}$-bundle. Thus, we get a smooth map $X \times_{G^{\prime}} U \rightarrow X \times_{G} U$ with fibers $G / G^{\prime}$. But the fiber 


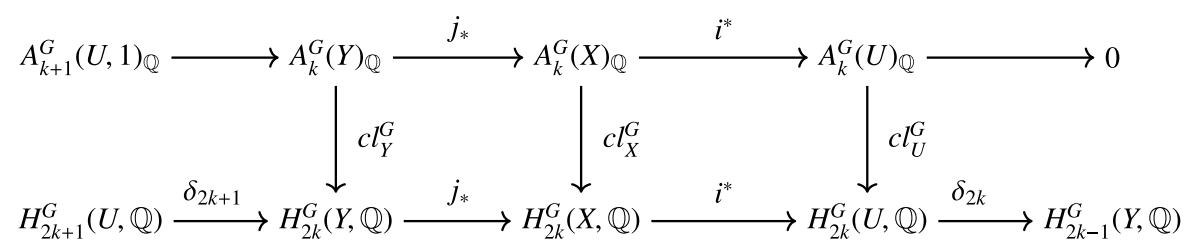

Figure 1. The $G$-equivariant localization diagram for $(Y, X, U)$.

$G / G^{\prime}$ is an affine space; therefore, $A_{*}^{G}(X)=A_{*}^{G^{\prime}}(X)$. Also by the same argument, we see that $H_{*}^{G}(X, \mathbb{Q})=H_{*}^{G^{\prime}}(X, \mathbb{Q})$. From now on, we will assume that $G$ is a reductive group.

Let $Y$ be a closed $G$-orbit, and let $U$ denote its complement in $X$. We proceed to analyze the equivariant cycle maps for $U$ and $X$. We claim that these maps are isomorphisms. Note that the number of $G$-orbits in $U$ is one less than the number of $G$-orbits in $X$, so we will use induction on the number of orbits. The base case, where there is a single $G$-orbit, is taken care of by Remark 7. To finish the inductive argument, we will use the $G$-equivariant localization diagram (Figure 1) for $(Y, X, U)$.

From the commuting of the squares in the diagram, we see that the long exact sequence of equivariant (higher) Chow groups splits at the third term and the long exact sequence of equivariant Borel-Moore homology groups splits into short exact sequences. In particular, we see from these splittings the isomorphisms

$$
A_{*}^{G}(X)_{\mathbb{Q}} \cong H_{*}^{G}(X, \mathbb{Q}) \text { and } H_{*}^{G}(X, \mathbb{Q}) \cong H_{*}^{G}(Y, \mathbb{Q}) \oplus H_{*}^{G}(U, \mathbb{Q}) .
$$

If $H$ is a closed subgroup of $G$, then the $H$-equivariant Borel-Moore homology of a point has the structure of a (graded) $H_{*}^{G}(p t, \mathbb{Q})$-module; therefore, for every $G$-orbit $Y^{\prime}$ in $X$, the same statement holds true for $H^{G}\left(Y^{\prime}, \mathbb{Q}\right)$. Note that we can reiterate these arguments for $H_{*}^{G}(U, \mathbb{Q})$ as well. Therefore, in light of the second isomorphism in $(8)$, we see that $H_{*}^{G}(X, \mathbb{Q})$ has a filtration, as a graded $H_{*}^{G}(p t, \mathbb{Q})$ module, given by the sums of the equivariant Borel-Moore homology groups of the $G$-orbits. Clearly, the associated graded module of this filtration is the direct sum of all $G$-equivariant Borel-Moore homology groups of the orbits of $X$. This finishes the proof.

\section{Proof of Theorem 2}

Our assumptions on $X$ and $G$ are as before; $X$ is a complex algebraic variety on which a connected complex linear algebraic group $G$ acts morphically with finitely many orbits. In addition, we assume the existence of a maximal torus $T$ 
of $G$ such that in every $G$-orbit in $X$, there exists a $T$-fixed point. Therefore, if $Z$ is a $G$-orbit in $X$, then $Z \cong G / K$ for some closed subgroup $K$ with $T \subset K$. In particular, $\operatorname{rk}(K)=\operatorname{rk}(G)$; hence, the odd rational cohomology of $Z$ is zero by [6, Corollary 12]. Below is a strengthening of this result.

LEMMA 9. If $Z$ denotes a $G$-orbit in $X$, then $Z$ has zero cohomology in odd degrees.

Proof. By our assumption, $Z$ is of the form $G / K$ for some closed subgroup $K$ with $T \subset K$. Observe that to prove our claim, it suffices to prove it for $G / K^{0}$, where $K^{0}$ is the connected component of $K$ containing the identity. Indeed, $G / K$ is the quotient of $G / K^{0}$ by the finite group $K / K^{0}$.

Now, since $T$ is connected, $T \subset K^{0}$; hence, we have a fibration $K^{0} / T \rightarrow$ $G / T \rightarrow G / K^{0}$. It is not difficult to see that the cohomology ring of $K^{0} / T$ is equal to the cohomology ring of the full flag variety of $K^{0}$, which is well known to be generated by the Chern classes of line bundles associated with characters of $T$. In particular, we see that these line bundles on $K^{0} / T$ extend to $G / T$; hence, we can apply the Leray-Hirsch theorem to conclude that

$$
H^{*}(G / T) \cong H^{*}\left(K^{0} / T\right) \otimes H^{*}\left(G / K^{0}\right)
$$

as graded $\mathbb{Z}$-modules. Since $G / T$ has zero cohomology in odd degrees, the same statement holds true for the cohomologies of $K^{0} / T$ and $G / K^{0}$. This finishes the proof.

Proof of Theorem 2. We continue with the notation of Lemma 9, that is, $Z=$ $G / K$ with $T \subset K$. Since all odd cohomology groups of $Z$ are zero, the Leray spectral sequence of the universal bundle $E G \times_{G} Z \rightarrow B G$ degenerates at the second page; hence, the rational equivariant cohomology of $Z$ is a free module over $H^{*}(B G, \mathbb{Q})=H_{G}^{*}(p t, \mathbb{Q})$. As this property holds true for every $G$-orbit in $X$, we conclude that $H_{*}^{G}(X, \mathbb{Q})$ is a free module over $H_{G}^{*}(p t, \mathbb{Q})$. Thus, by tensoring the filtration (from Theorem 1) of the equivariant Borel-Moore homology of $X$ with $\mathbb{Q}=H_{G}^{*}(p t, \mathbb{Q}) /\left(S_{+}^{W}\right)$, we finish the proof of our theorem.

\section{Applications}

5.1. Proof of Theorem 3. Let $X$ be an irreducible $G$-scheme, and let $X_{0}$ denote the open $G$-orbit in $X$. We will call $X \mathrm{CH}$-congruent (Chow group/rational homology congruent) if the cycle map to the rational cohomology induced via the Poincare duality, that is $c l_{X_{0}}: A^{*}\left(X_{0}\right)_{\mathbb{Q}} \rightarrow H^{*}\left(X_{0}, \mathbb{Q}\right)$, is an isomorphism. 


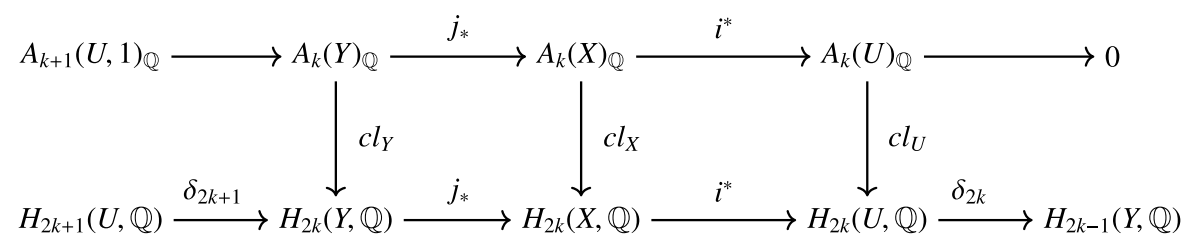

Figure 2. The nonequivariant localization diagram for $(Y, X, U)$.

More generally, we will call a $G$-scheme $X \mathrm{CH}$-congruent if each irreducible component of $X$ is $\mathrm{CH}$-congruent.

Let $H$ be a connected reductive subgroup of a connected reductive group $G$. Let $T_{H}$ be a maximal torus of $H$ and let $T$ be a maximal torus of $G$ containing $T_{H}$. We will denote by $W_{H}$ and $W$ the Weyl groups of $\left(H, T_{H}\right)$ and $(G, T)$, respectively. We denote by $S_{H}$ and $S$ the symmetric $\mathbb{Q}$-algebras over the character groups of $T_{H}$ and $T$, respectively. Finally, we will denote by $\left(S_{+}^{W}\right)$ the ideal generated by the restriction to $S_{H}^{W_{H}}$ of the homogeneous positive degree $W$-invariants in $S$.

LEMMA 10. Let $H$ be a closed, connected, and reductive subgroup of a connected reductive group $G$. Then the following are equivalent:

(1) The rational cohomology of $G / H$ vanishes in odd degree.

(2) The ranks of $G$ and $H$ are the same.

(3) The homogeneous space $G / H$ is $C H$-congruent.

Moreover, $A^{*}(G / H)_{\mathbb{Q}} \cong S_{H}^{W_{H}} /\left(S_{+}^{W}\right)$ and $A_{T}^{*}(G / H)_{\mathbb{Q}} \cong S \otimes_{S^{W}}\left(S_{+}^{W}\right)$.

Proof. See [6, Corollary 12].

As before, for every closed embedding $j: Y \hookrightarrow X$ and the complementary embedding $i: U \hookrightarrow X$, where $U:=X \backslash Y$, we have a localization diagram (Figure 2) as in Figure 1.

We will make use of the above (nonequivariant) diagram in the context of equivariant embeddings of $\mathrm{CH}$-congruent spaces. Let $X$ be a smooth complete spherical embedding of a $\mathrm{CH}$-congruent homogeneous space $G / H$. Under these assumptions, we know that $X$ has a cellular decomposition via the BiałynickiBirula decomposition. Let $Y$ denote the complement of $U:=G / H$ in $X$ and let $i: U \hookrightarrow X$ and $j: Y \rightarrow X$ denote the corresponding inclusions. Since $U$ is a $\mathrm{CH}$-congruent homogeneous space, the long exact sequences in Figure 2 break down into short exact sequences. Indeed, the vertical arrows $c l_{U}$ and $c l_{X}$ 
are isomorphisms. On both the top and the bottom sequences, the maps $i^{*}$ 's are induced from the restriction map to the open set $U$, and, therefore, they are actually equivalent maps. (So, in this case, our notation is unambiguous.) It follows that, by the exactness of the Chow group sequence, $i^{*}$ is surjective; hence, $\delta_{2 k}$ is the 0 map. Note that $U$ is a $\mathrm{CH}$-congruent variety, so its odd (co)homology vanishes; therefore, $\delta_{2 k+1}$ is the 0 map as well. It follows that $H_{2 k+1}(Y, \mathbb{Q}) \cong H_{2 k+1}(X, \mathbb{Q})$.

By the exactness of the Chow sequence, and by identifying $i^{*}$ 's in the top and the bottom sequences, we see that the images of $j_{*}$ 's are isomorphic subgroups. Furthermore, we know that the bottom $j_{*}$ is injective since $\delta_{2 k+1}=0$. However, we do not claim that the $j_{*}$ 's are the 'same' (yet) since we do not know if $c l_{Y}$ is an isomorphism or not. It turns out that this is the case by a deep result due to Totaro and Jannsen.

According to [16], a linear scheme is a scheme which can be obtained by an inductive procedure starting with an affine space of any dimension, in such a way that the complement of a linear scheme embedded in the affine space is also a linear scheme, and a scheme which can be stratified as a finite disjoint union of linear schemes is a linear scheme.

LEMMA 11. For any complex linear scheme $Z$, the natural map

$$
A_{i}(Z)_{\mathbb{Q}} \longrightarrow W_{-2 i} H_{2 i}(Z, \mathbb{Q})
$$

from the rational Chow group into the smallest subspace of rational Borel-Moore homology with respect to the weight filtration is an isomorphism.

Proof. See [16, Theorem 3].

REMARK 12. (1) A finite union of linear schemes is a linear scheme.

(2) Any spherical variety is linear; see the addendum at the end of Section 2 in [16].

(3) The complement of the open $G$-orbit in a spherical variety is a linear scheme since it is a union of finitely many $G$-orbit closures.

Now, by Remark 12 and Lemma 11, we know that the vertical map $c l_{Y}$ is injective. Since the bottom $j_{*}$ is injective and it has the same image as the top $j_{*}$, we see that the top $j_{*}$ has to be injective as well. It follows that the localization long exact sequence for the higher Chow groups breaks down. These observations give us the following result. 
THEOREM 13. Let $X$ be a smooth complete spherical $G$-variety. If the open $G$ orbit, $\mathrm{G} / \mathrm{H}$ is $\mathrm{CH}$-congruent, then we have

$$
H_{2 k}(X-G / H, \mathbb{Q}) \cong A_{k}(X-G / H)_{\mathbb{Q}} \text { for all } k \geqslant 0,
$$

and

$$
A_{k}(X)_{\mathbb{Q}} \cong A_{k}(X-G / H)_{\mathbb{Q}} \oplus A_{k}(G / H)_{\mathbb{Q}} \text { for all } k \geqslant 0 .
$$

Furthermore, we have $H_{2 k+1}(X, \mathbb{Q})=H_{2 k+1}(X-G / H, \mathbb{Q})=\{0\} \quad$ for all $k \geqslant 0$.

Proof. Let $U$ denote the open $G$-orbit, $U=G / H$, and let $Y$ denote its complement, $X-U$. By the above discussion, it suffices to show the last assertion that, for all $k \in \mathbb{Z}_{\geqslant 0}$, the groups $H_{2 k+1}(Y, \mathbb{Q}) \cong H_{2 k+1}(X, \mathbb{Q})$ are trivial. But this follows from the fact that the Borel-Moore homology is equal to the singular homology for an oriented compact manifold and the fact that $X$ has a cellular decomposition, where the cells are complex affine spaces. Indeed, $X$ has a Białynicki-Birula decomposition since it is a smooth complete spherical variety; hence, the odd homology of $X$ vanishes.

Let $H$ be a closed subgroup of $G$ such that the subset $B H$ is open in $G$. In this case, $H$ is called a spherical subgroup and the homogeneous space $G / H$ is called a spherical homogeneous space.

Let $G / H$ be a spherical homogeneous space. A spherical embedding $X$ of $G / H$ is called toroidal if $X$ has no $B$-invariant divisor which is $G$-invariant. $X$ is called simple if it has a unique closed $G$-orbit. Wonderful varieties are toroidal as shown by Bien and Brion in [2]. In fact, any smooth complete toroidal spherical variety is a wonderful variety. The stabilizers of generic points in $G$-orbits of a simple toroidal embedding are recently looked at by Batyrev and Moreau in [1].

LEMMA 14. Let $X$ be a simple toroidal spherical embedding of $G / H$ corresponding to an uncolored cone $\sigma$. Denote by $I(\sigma)$ the set of all spherical roots in $\mathcal{S}$ that vanish on $\sigma$. Let $x^{\prime}$ be a point in the unique closed $G$-orbit $X^{\prime}$ of $X$ and $\operatorname{Iso}_{G}\left(x^{\prime}\right)$ the isotropy subgroup of $x^{\prime}$ in $G$. Then, up to a conjugation, we have the inclusions:

$$
H_{I(\sigma)} \subset \operatorname{Iso}_{G}\left(x^{\prime}\right) \subset N_{G}\left(H_{I(\sigma)}\right) .
$$

Moreover, there is a homomorphism from $N_{G}\left(H_{I(\sigma)}\right)$ to $\operatorname{Hom}\left(\sigma^{\perp} \cap M, \mathbb{C}^{*}\right)$ whose kernel is $\operatorname{Iso}_{G}\left(x^{\prime}\right)$.

Proof. See [1, Theorem 1.2]. 
The subgroups $H_{I(\sigma)}$ in Lemma 14 are called the satellites of $H$. For a closed subgroup $K$ of $G$, let $K^{\text {red }}$ denote the maximal reductive subgroup of $K$.

REMARK 15. In [1, Lemma 7.2], Batyrev and Moreau proved the following result, which they attribute to Brion and Peyre [7]: if $I$ is a subset of the spherical roots $\mathcal{S}$ of $G / H$, then $H_{I}^{\text {red }} \subset H$.

Proposition 16. Let $H$ be a spherical subgroup of $G$ of maximal rank, that is, $\operatorname{rk}(H)=\operatorname{rk}(G)$. We assume that $G / H$ has a smooth complete toroidal embedding, denoted by $X$. Let $X_{i}$ be a $G$-orbit closure in $X$. If $H_{i}$ is the stabilizer of a generic point in $X_{i}$, that is, $H_{i}=\operatorname{Stab}_{G}(x)$ for some $x$ in the open $G$-orbit in $X_{i}$, then the rank of $H_{i}$ is equal to the rank of $G$. Furthermore, we have the isomorphisms $H_{k}\left(X_{i}-G / H_{i}, \mathbb{Q}\right) \cong A_{k}\left(X_{i}-G / H_{i}\right)_{\mathbb{Q}}$ and $A_{k}\left(X_{i}\right)_{\mathbb{Q}} \cong A_{k}\left(X_{i}-G / H_{i}\right)_{\mathbb{Q}} \oplus$ $A_{k}\left(G / H_{i}\right)_{\mathbb{Q}}$ for all $k \geqslant 0$.

Proof. Since $X$ is a wonderful variety, any $G$-orbit closure $X_{i}$ is smooth, and, furthermore, $X_{i}$ is a wonderful variety itself. Therefore, in light of Theorem 13, our second claim will follow from the first one, that is, the rank of the stabilizer of a generic point in $X_{i}$ is equal to $\operatorname{rk}(H)=\operatorname{rk}(G)$.

Note that the closed $G$-orbit in $X_{i}$ is the closed $G$-orbit in $X$. Let $G / Q$ denote this closed $G$-orbit, where $Q$ is a parabolic subgroup. The satellite of $H$ corresponding to $G / Q$ is $H_{\emptyset}=Q$. It follows from Remark 15 that the Levi subgroup $L_{Q}$ of $Q$ is contained in the stabilizer subgroup in $G$ of a generic point in $X_{i}$. Since the $\operatorname{rank} \operatorname{rk}\left(L_{Q}\right)$ of $L_{Q}$ is equal to the rank of $G$, the proof of our proposition is complete.

We are now ready to prove our third main result.

Proof of Theorem 3. We will apply Theorem 2. In the course of the proof of Proposition 16, we showed that for every $G$-orbit $Y_{i}$ in $X$, the stabilizer of a generic point from $Y_{i}$ contains the Levi subgroup $L_{Q}$ of the parabolic subgroup $Q$, where $G / Q$ is the closed orbit in $X$. Since $T \subset L_{Q}$, we see that every $G$-orbit in $X$ has a $T$-fixed point. This finishes the proof.

5.2. Proof of Theorem 4. Let $G$ be a connected reductive algebraic group and let $T$ and $B$ denote a maximal torus and a Borel subgroup such that $T \subset B \subset G$. Let $I$ and $J$ be two subsets from the set of simple roots $\Delta=\Delta(G, B, T)$. A double flag variety is a product of the form $X:=G / P_{I} \times G / P_{J}$, where $P_{I}$ and $P_{J}$, respectively, are the standard parabolic subgroups determined by the subsets $I$ and $J$. Starting with the work of Littelmann in [12], it is understood that the cones over these varieties can be used for calculating the multiplicities in the 
tensor products of irreducible representations of $G$. For a good exposition of this idea, see [14, Section 2.11].

The diagonal $G$-action on a double flag variety $X$ is given by $g \cdot\left(a P_{I}, b P_{J}\right)=$ $\left(g a P_{I}, g b P_{J}\right)$ for $g, a, b \in G$. The canonical projection $\pi: X \rightarrow G / P_{J}$ is $G$ equivariant and it turns $X$ into a homogeneous fiber bundle over $G / P_{J}$ with fiber $G / P_{I}$ at every point $g P_{J}(g \in G)$ of the base $G / P_{J}$. To distinguish it from the other fibers, let us denote by $Y$ the fiber $G / P_{I}$ at the 'origin' $e P_{J}$ of $G / P_{J}$. Then any $G$-orbit in $X$ meets $Y$. Note also that if $g \cdot y \in Y$ for some $g \in G$ and $y \in X$, then $g \in P_{I}$. This means that, for every $G$-orbit in $X$, there exists a unique $P_{I}$-orbit in $G / P_{J}$, which is viewed as a fiber of $\pi$ at $e P_{J}$. It is well known that these $P_{I}$-orbits are in 1-1 correspondence with $\left(W_{I}, W_{J}\right)$-double cosets in $W$, where $W_{I}$ and $W_{J}$, respectively, are the subgroups of $W$ determined by $I$ and $J$; see [3, Section 21.16]. In fact, every $P_{I}$-orbit in $G / P_{J}$ is of the form $P_{I} \cdot \dot{w}$, where $\dot{w} \in N_{G}(T)$ and $w \in W$ is a minimal length representative of a double coset $W_{I} w W_{J}$ in $W$.

Let us denote by ${ }^{I} W^{J}$ the set of minimal coset representatives of the $\left(W_{I}, W_{J}\right)$ double cosets in $W$. It follows from the above discussion that the set of $G$-orbits in $X$ is finite as it is in bijection with ${ }^{I} W^{J}$. Furthermore, each $G$-orbit in $X$ is of the form $G \cdot \dot{w} \cong G / \operatorname{Stab}_{G}(\dot{w})$ for some $w \in{ }^{I} W^{J}$. Note that the stabilizer subgroup of any element of $W$ is a standard parabolic subgroup in $G$; therefore, $T \subset \operatorname{Stab}_{G}(\dot{w})$.

We are now ready to prove the final result of our paper.

Proof of Theorem 4. We already know that the $G$-orbits in the double flag variety $X=G / P_{I} \times G / P_{J}$ are parametrized by ${ }^{I} W^{J}$. It follows from the above discussion that every $G$-orbit in $X$ contains a $T$-fixed point. By the Künneth formula, the (integral) cohomology ring of $X$ is isomorphic to the tensor product of the (integral) cohomology rings $H^{*}\left(G / P_{I}\right)$ and $H^{*}\left(G / P_{J}\right)$. Therefore, after tensoring with $\mathbb{Q}$, Theorem 2 gives us the $\mathbb{Q}$-vector space decomposition in $H^{*}\left(G / P_{I}, \mathbb{Q}\right) \otimes H^{*}\left(G / P_{J}, \mathbb{Q}\right) \cong \bigoplus_{w \in^{I} W^{J}} H^{*}\left(G / \operatorname{Stab}_{G}(\dot{w}), \mathbb{Q}\right)$.

The complexity of a group action $G \times X \rightarrow X$ is defined as the minimal codimension of a Borel orbit in general position. A $G$-variety $X$ is spherical if and only if $X$ is normal and the complexity of the action is zero; see [14, Section 3.15]. For $G:=\mathbf{S L}_{n}$, the structures of the posets of $G$-orbit closures in the double flag varieties of complexity $\leqslant 1$ are determined in $[8,9]$. This information can be used for further studying the decompositions described in Theorem 4.

\section{Acknowledgements}

The second author is partially supported by a grant from Louisiana Board of Regents. We thank Slawomir Kwasik and Özlem Uğurlu for useful conversations. 
We are grateful to Michel Brion for his constructive criticisms and comments. Finally, we thank the referee for her/his comments and suggestions which improved the quality of our paper.

\section{References}

[1] V. Batyrev and A. Moreau, 'Satellites of spherical subgroups', Preprint, 2016, ArXiv e-prints.

[2] F. Bien and M. Brion, 'Automorphisms and local rigidity of regular varieties', Compos. Math. 104(1) (1996), 1-26.

[3] A. Borel, Linear Algebraic Groups, 2nd edn, Graduate Texts in Mathematics, 126, (Springer, New York, 1991).

[4] M. Brion, 'Quelques propriétés des espaces homogènes sphériques', Manuscripta Math. 55(2) (1986), 191-198.

[5] M. Brion, 'Equivariant Chow groups for torus actions', Transform. Groups 2(3) (1997), 225-267.

[6] M. Brion, 'Equivariant cohomology and equivariant intersection theory', in Representation Theories and Algebraic Geometry (Montreal, PQ, 1997), NATO Adv. Sci. Inst. Ser. C Math. Phys. Sci., 514 (Kluwer Academic Publishers, Dordrecht, 1998), 1-37. Notes by Alvaro Rittatore.

[7] M. Brion and E. Peyre, 'The virtual Poincaré polynomials of homogeneous spaces', Compos. Math. 134(3) (2002), 319-335.

[8] M. B. Can, 'The cross-section of a spherical double cone', Adv. Appl. Math. 101 (2018), 215-231.

[9] M. B. Can and L. Tien, 'Diagonal orbits in a double flag variety of complexity one, type A', Preprint, 2018, arXiv:1810.06513.

[10] D. Edidin and W. Graham, 'Characteristic classes in the Chow ring', J. Algebraic Geom. 6(3) (1997), 431-443.

[11] D. Edidin and W. Graham, 'Equivariant intersection theory', Invent. Math. 131(3) (1998), 595-634.

[12] P. Littelmann, 'On spherical double cones', J. Algebra 166(1) (1994), 142-157.

[13] D. Luna and T. Vust, 'Plongements d'espaces homogènes', Comment. Math. Helv. 58(2) (1983), 186-245.

[14] D. A. Timashev, Homogeneous Spaces and Equivariant Embeddings, Encyclopaedia of Mathematical Sciences, 138 (Springer, Heidelberg, 2011), Invariant Theory and Algebraic Transformation Groups, 8.

[15] B. Totaro, 'The Chow ring of a classifying space', in Algebraic K-theory (Seattle, WA, 1997), Proc. Sympos. Pure Math., 67 (American Mathematical Society, Providence, RI, 1999), 249-281.

[16] B. Totaro, 'Chow groups, Chow cohomology, and linear varieties', Forum Math. Sigma 2(e17) (2014), 25 pages.

[17] È. B. Vinberg, 'Complexity of actions of reductive groups', Funktsional. Anal. i Prilozhen. 20(1) (1986), 1-13. 96. 